\title{
SOLUTION OF TIME-VARYING SINGULAR NONLINEAR SYSTEMS BY SINGLE-TERM WALSH SERIES
}

\author{
B. SEPEHRIAN AND M. RAZZAGHI
}

Received 6 February 2002

A method for finding the solution of time-varying singular nonlinear systems by using single-term Walsh series is proposed. The properties of single-term Walsh series are given and are utilized to find the solution of time-varying singular nonlinear systems.

\section{Introduction}

Singular nonlinear systems has been of interest to some investigators $[4,12]$, however no closed-form solution was given in $[4,12]$. In some analysis of neural networks, both singular systems [8] and bilinear systems [16] have been used. For singular bilinear systems, Lewis et al. [11] applied the Walsh function (WF) approach for time-invariant singular bilinear systems and Hsiao and Wang [9] used the Haar wavelets for the solution of time-varying singular nonlinear systems.

Walsh functions (WFs) have received considerable attention in dealing with various problems of dynamic systems. Chen and Hsiao $[5,6,7]$ applied the WF technique to the analysis, optimal control, and synthesis of linear systems. WFs have also found wide applications in signal processing, communication, and pattern recognition [13]. Rao et al. [14] presented a method of extending computation beyond the limit of the initial normal interval in Walsh series analysis of dynamical systems. In [14] various time functions in the system were first expanded in terms of their truncated WF with unknown coefficients. Using the Kronecker product [10], the unknown coefficient of the rate variable was obtained by finding the inverse of a square matrix. It was shown that this method involve some numerical difficulties if the dimension of this matrix is large. To remove the inconveniences in WF technique, the single-term Walsh series (STWS) was introduced in [14], and Balachandran and Murugesan [1,2,3] applied STWS technique to the analysis of the linear and nonlinear singular systems. The STWS method provides block-pulse and discrete solutions to any length of time.

In the present paper, we use the STWS approach for the solution of time-varying singular nonlinear systems. As compared to [9], our method is simpler and consumes less computer time. 
The paper is organized as follows: in Section 2 we describe the basic properties of the WF and STWS required for our subsequent development. Section 3 is devoted to the formulation of the time-varying singular nonlinear systems. In Section 4 we apply the proposed numerical method to the time-varying singular nonlinear systems and in Section 5, we report our numerical finding and demonstrate the accuracy of the proposed method.

\section{Properties of WF and STWS}

2.1. Walsh functions. A function $f(t)$, integrable in $[0,1)$, may be approximated using WF as

$$
f(t)=\sum_{i=0}^{\infty} f_{i} \phi_{i}(t)
$$

where $\phi_{i}(t)$ is the $i$ th WF and $f_{i}$ is the corresponding coefficient. In practice, only the first $m$ terms are considered, where $m$ is an integral power of 2 . Then from (2.1), we get

$$
f(t)=\sum_{i=0}^{m-1} f_{i} \phi_{i}(t)=F^{T} \Phi(t),
$$

where

$$
F=\left(f_{0}, f_{1}, \ldots, f_{m-1}\right)^{T}, \quad \Phi(t)=\left(\phi_{0}(t), \phi_{1}(t), \ldots, \phi_{m-1}(t)\right)^{T}
$$

The coefficients $f_{i}$ are chosen to minimize the mean integral square error

$$
\epsilon=\int_{0}^{1}\left(f(t)-F^{T} \Phi(t)\right)^{2} d t
$$

and are given by

$$
f_{i}=\int_{0}^{1} f(t) \phi_{i}(t) d t
$$

The integration of the vector $\Phi(t)$ defined in (2.3) can be approximated by

$$
\int_{0}^{t} \Phi\left(t^{\prime}\right) d t^{\prime} \simeq E \Phi(t),
$$

where $E$ is the $m \times m$ operational matrix for integration with $E_{1 \times 1}=1 / 2$ and is given in [16].

2.2. Single-term Walsh series. With the STWS approach, in the first interval, the given function is expanded as STWS in the normalized interval $\tau \in[0,1)$, which corresponds to $t \in[0,1 / m)$ by defining $\tau=m t, m$ being any integer. In STWS, the matrix $E$ in (2.6) becomes $E=1 / 2$. 
Let $\dot{x}(\tau)$ and $x(\tau)$ be expanded by STWS series in the first interval as

$$
\dot{x}(\tau)=V^{(1)} \phi_{0}(\tau), \quad x(\tau)=X^{(1)} \phi_{0}(\tau),
$$

and in the $k$ th interval as

$$
\dot{x}(\tau)=V^{(k)} \phi_{0}(\tau), \quad x(\tau)=X^{(k)} \phi_{0}(\tau) .
$$

Integrating (2.7) with $E=1 / 2$, we get

$$
X^{(1)}=\frac{1}{2} V^{(1)}+x(0)
$$

where $x(0)$ is the initial condition. According to Sannuti [15], we have

$$
V^{(1)}=\int_{0}^{1} \dot{x}(\tau) d \tau=x(1)-x(0)
$$

In general, for any interval $k, k=1,2, \ldots$, we obtain

$$
\begin{aligned}
& X^{(k)}=\frac{1}{2} V^{(k)}+x(k-1), \\
& x(k)=V^{(k)}+x(k-1) .
\end{aligned}
$$

In (2.11) and (2.12), $X^{(k)}$ and $x(k)$ give the block-pulse and the discrete values of the state, respectively.

\section{Problem statement}

Consider a time-varying singular nonlinear system of the following form:

$$
E(t) \dot{x}(t)=f(t, x(t), u(t)), \quad x(0)=x_{0},
$$

where the singular matrix $E(t) \in \mathbb{R}^{n \times n}$, the nonlinear function $f \in \mathbb{R}^{n}$, the state $x(t) \in \mathbb{R}^{n}$, and the control $u(t) \in \mathbb{R}^{q}$. The response $x(t)$ is required to be found.

\section{Solution of time-varying singular nonlinear systems via STWS}

Normalizing (3.1) by defining $\tau=m t$, we get

$$
m E(\tau) \dot{x}(\tau)=f(\tau, x(\tau), u(\tau)), \quad x(0)=x_{0} .
$$




\section{STWS for singular nonlinear systems}

Let $E(\tau)$ be expressed by STWS in the $k$ th interval as

$$
E(\tau)=E^{(k)} \phi_{0}(\tau)
$$

where $E^{(k)} \in \mathbb{R}^{n \times n}$. By using (2.8) and (2.11), we get

$$
x(\tau)=\left(\frac{1}{2} V^{(k)}+x(k-1)\right) \phi_{0}(\tau) .
$$

To solve (4.1), we first substitute (4.3) in $f(\tau, x(\tau), u(\tau))$; we then express the resulting equation by STWS as

$$
f\left(\tau,\left(\frac{1}{2} V^{(k)}+x(k-1)\right) \phi_{0}(\tau), u(\tau)\right)=F^{(k)} \phi_{0}(\tau)
$$

Using (4.1), (4.2), (4.3), and (4.4), we get

$$
m E^{(k)} V^{(k)}=F^{(k)} .
$$

By solving (4.5), the components of $V^{(k)}$ can be obtained. By substituting $V^{(k)}$ in (2.11) and (2.12), we obtain block-pulse and discrete approximations of the state, respectively. Further, using (2.7), we get

$$
x(\tau)=\int_{0}^{\tau} \dot{x}\left(\tau^{\prime}\right) d \tau^{\prime}+x(0)=V^{(1)} \tau+x(0) .
$$

Thus, we can obtain a continuous approximation of the state as

$$
x(\tau)=V^{(k)} \tau+x(k-1) .
$$

\section{Numerical examples}

Three examples are given in this section. These examples were considered by Hsiao and Wang [9] by using Haar wavelets. Our method differs from their approach and thus these examples could be used as a basis for comparison.

Example 5.1. Consider a time-varying nonlinear singular system of the following form [9]:

$$
\left[\begin{array}{ccc}
0 & 1 & 0 \\
0 & 0 & t^{2} \\
0 & 0 & 0
\end{array}\right] \dot{x}(t)=\left[\begin{array}{c}
t x_{1}(t)+x_{2}(t) \\
\exp (t) x_{1}(t) x_{2}(t) \\
x_{2}(t)\left(x_{1}(t)+x_{3}(t)\right)
\end{array}\right]+\left[\begin{array}{c}
0 \\
2 t^{2} \exp (-t) \\
0
\end{array}\right], \quad x(0)=\left[\begin{array}{c}
2 \\
0 \\
-2
\end{array}\right] .
$$

The exact solution is (see [9])

$$
x(t)=\left[\begin{array}{c}
2 \exp (-t)(1-2 t) \\
t^{2} \exp (-t) \\
-2 \exp (-t)(1-2 t)
\end{array}\right] .
$$




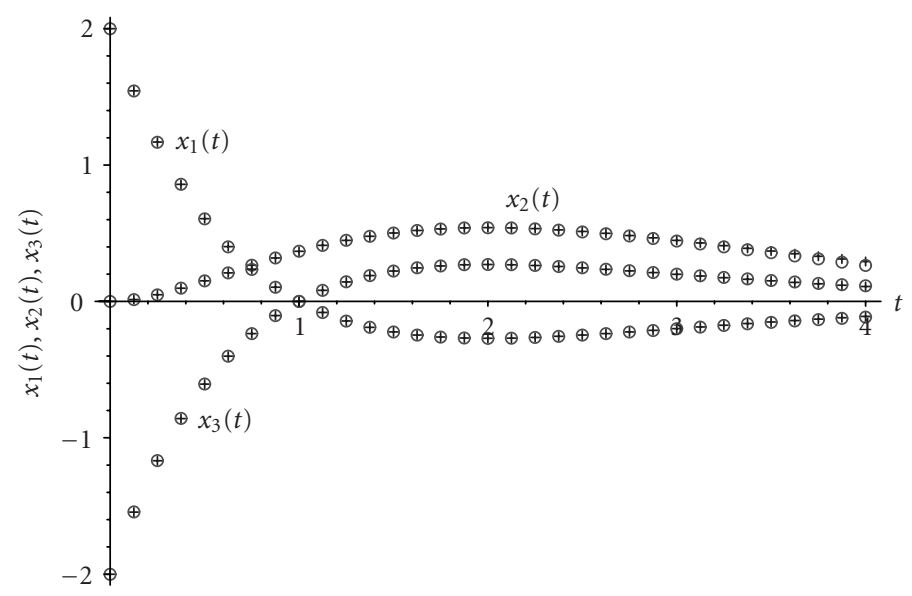

Figure 5.1. STWS with $m=32$ (circles) and exact solution: ++++ of Example 5.1.

To solve (5.1) by STWS, we first express $t, t^{2}, \exp (t)$, and $2 t^{2} \exp (-t)$ by STWS, then we substitute these values together with $V_{i}^{(k)}=\dot{x}_{i}(t)$ and $x_{i}(t)=(1 / 2) V_{i}^{(k)}+x_{i}(k-1)$, $i=1,2,3$, in (5.1). By solving the resulting equation, $V^{(k)}=\left[V_{1}^{(k)}, V_{2}^{(k)}, V_{3}^{(k)}\right]^{T}$ can be calculated. By using (2.11), (2.12), and (4.7), block-pulse, discrete, and continuous approximations of state $x(t)$ are obtained.

The comparison between STWS solution with $m=32$ and the exact solution for $t \in$ $[0,4)$ is shown in Figure 5.1.

Example 5.2. Consider the following time-invariant singular nonlinear system [9]:

$$
\left[\begin{array}{lllll}
1 & 0 & 0 & 0 & 0 \\
0 & 0 & 0 & 0 & 0 \\
0 & 0 & 0 & 0 & 0 \\
0 & 0 & 0 & 0 & 0 \\
0 & 0 & 0 & 0 & 0
\end{array}\right] \dot{x}(t)+\left[\begin{array}{c}
x_{3}(t)-x_{5}(t) \\
x_{2}(t)+x_{3}(t)-x_{4}(t)-x_{5}(t) \\
\left(x_{1}(t)+x_{2}(t)-1\right)^{2}-x_{3}(t) \\
-x_{4}(t) \\
x_{2}(t)\left(x_{1}(t)+x_{2}(t)\right)-x_{5}(t)
\end{array}\right]=\left[\begin{array}{l}
0 \\
1 \\
0 \\
0 \\
0
\end{array}\right], \quad x(0)=\left[\begin{array}{l}
0 \\
0 \\
1 \\
0 \\
0
\end{array}\right] .
$$

The results obtained by STWS with $m=16$ and $m=110$ together with those obtained by Haar wavelets with $m=512$ are presented in Tables 5.1, 5.2, and 5.3, respectively.

Example 5.3. Consider a time-invariant nonlinear singular system of the following form [9]:

$$
\left[\begin{array}{lll}
0 & 1 & 0 \\
0 & 0 & 0 \\
0 & 0 & 0
\end{array}\right] \dot{x}(t)+\left[\begin{array}{ccc}
0 & 0 & 1 \\
1 & 1 & 0 \\
1 & 0 & -3
\end{array}\right] x(t)+\left[\begin{array}{c}
0 \\
0 \\
x_{3}^{3}(t)
\end{array}\right]=\left[\begin{array}{l}
0 \\
1 \\
0
\end{array}\right], \quad x(0)=\left[\begin{array}{c}
2 \\
-1 \\
-2
\end{array}\right] .
$$

The results obtained by STWS with $m=24$ and $m=32$ and those obtained by Haar wavelets with $m=32$ and $m=128$ are presented in Tables 5.4 and 5.5, respectively. 
134 STWS for singular nonlinear systems

Table 5.1. Estimated values of $x_{1}(t), x_{2}(t), x_{3}(t), x_{4}(t)$, and $x_{5}(t)$ by STWS with $m=16$.

\begin{tabular}{c|ccccc}
\hline Time & \multicolumn{1}{c}{$x_{1}(t)$} & $x_{2}(t)$ & $x_{3}(t)$ & $x_{4}(t)$ & $x_{5}(t)$ \\
\hline 0.0 & 0.0000 & 0.0000 & 1.0000 & 0.0000 & 0.0000 \\
0.5 & -0.3297 & 0.5771 & 0.5671 & 0.0000 & 0.1443 \\
1.0 & -0.4776 & 0.8003 & 0.4596 & 0.0000 & 0.2599 \\
1.5 & -0.5486 & 0.9023 & 0.4186 & 0.0000 & 0.3209 \\
2.0 & -0.5835 & 0.9514 & 0.4004 & 0.0000 & 0.3516 \\
2.5 & -0.6008 & 0.9755 & 0.3919 & 0.0000 & 0.3674 \\
3.0 & -0.6094 & 0.9875 & 0.3877 & 0.0000 & 0.3752 \\
3.5 & -0.6137 & 0.9935 & 0.3856 & 0.0000 & 0.3791 \\
4.0 & -0.6159 & 0.9965 & 0.3846 & 0.0000 & 0.3811 \\
\hline
\end{tabular}

Table 5.2. Estimated values of $x_{1}(t), x_{2}(t), x_{3}(t), x_{4}(t)$, and $x_{5}(t)$ by STWS with $m=110$.

\begin{tabular}{c|ccccc}
\hline Time & $x_{1}(t)$ & $x_{2}(t)$ & $x_{3}(t)$ & $x_{4}(t)$ & $x_{5}(t)$ \\
\hline 0.0 & 0.0000 & 0.0000 & 1.0000 & 0.0000 & 0.0000 \\
0.5 & -0.3295 & 0.5774 & 0.5658 & 0.0000 & 0.1431 \\
1.0 & -0.4775 & 0.8006 & 0.4582 & 0.0000 & 0.2587 \\
1.5 & -0.5485 & 0.9027 & 0.4171 & 0.0000 & 0.3197 \\
2.0 & -0.5834 & 0.9518 & 0.3989 & 0.0000 & 0.3507 \\
2.5 & -0.6007 & 0.9760 & 0.3903 & 0.0000 & 0.3663 \\
3.0 & -0.6094 & 0.9880 & 0.3861 & 0.0000 & 0.3741 \\
3.5 & -0.6137 & 0.9940 & 0.3841 & 0.0000 & 0.3780 \\
4.0 & -0.6159 & 0.9970 & 0.3830 & 0.0000 & 0.3800 \\
\hline
\end{tabular}

Table 5.3. Estimated values of $x_{1}(t), x_{2}(t), x_{3}(t), x_{4}(t)$, and $x_{5}(t)$ by Haar wavelets with $m=512$.

\begin{tabular}{c|ccccc}
\hline Time & $x_{1}(t)$ & $x_{2}(t)$ & $x_{3}(t)$ & $x_{4}(t)$ & $x_{5}(t)$ \\
\hline 0.0 & 0.0000 & 0.0000 & 1.0000 & 0.0000 & 0.0000 \\
0.5 & -0.3295 & 0.5774 & 0.5658 & 0.0000 & 0.1431 \\
1.0 & -0.4775 & 0.8006 & 0.4582 & 0.0000 & 0.2587 \\
1.5 & -0.5485 & 0.9027 & 0.4171 & 0.0000 & 0.3197 \\
2.0 & -0.5834 & 0.9518 & 0.3989 & 0.0000 & 0.3507 \\
2.5 & -0.6007 & 0.9760 & 0.3903 & 0.0000 & 0.3663 \\
3.0 & -0.6094 & 0.9880 & 0.3861 & 0.0000 & 0.3741 \\
3.5 & -0.6137 & 0.9940 & 0.3841 & 0.0000 & 0.3780 \\
4.0 & -0.6159 & 0.9970 & 0.3830 & 0.0000 & 0.3800 \\
\hline
\end{tabular}


Table 5.4. Estimated values of $x_{1}(t), x_{2}(t)$, and $x_{3}(t)$ by STWS with $m=24$ and $m=32$.

\begin{tabular}{l|ccc|crc}
\hline \multirow{2}{*}{ Time } & \multicolumn{3}{|c|}{ STWS with $m=24$} & \multicolumn{3}{c}{ STWS with $m=32$} \\
& $x_{1}(t)$ & $x_{2}(t)$ & $x_{3}(t)$ & $x_{1}(t)$ & $x_{2}(t)$ & $x_{3}(t)$ \\
\hline 0.0 & 2.00000 & -1.00000 & -2.00000 & 2.00000 & -1.00000 & -2.00000 \\
0.125 & 1.75175 & -0.75175 & -1.97189 & 1.75175 & -0.75175 & -1.97189 \\
0.25 & 1.50706 & -0.50706 & -1.94309 & 1.50706 & -0.50706 & -1.94309 \\
0.375 & 1.26601 & -0.26601 & -1.91353 & 1.26601 & -0.26601 & -1.91353 \\
0.5 & 1.02871 & -0.02871 & -1.88316 & 1.02871 & -0.02871 & -1.88316 \\
0.625 & 0.79526 & 0.20474 & -1.85187 & 0.79526 & 0.20474 & -1.85187 \\
0.75 & 0.56578 & 0.43422 & -1.81960 & 0.56578 & 0.43422 & -1.81960 \\
0.875 & 0.34040 & 0.65960 & -1.78622 & 0.34040 & 0.65960 & -1.78622 \\
1.0 & 0.11927 & 0.88073 & -1.75160 & 0.11927 & 0.88073 & -1.75160 \\
\hline
\end{tabular}

Table 5.5. Estimated values of $x_{1}(t), x_{2}(t)$, and $x_{3}(t)$ by Haar wavelets with $m=32$ and $m=128$.

\begin{tabular}{l|ccc|ccc}
\hline \multirow{2}{*}{ Time } & \multicolumn{3}{|c|}{ Haar wavelets with $m=32$} & \multicolumn{3}{c}{ Haar wavelets with $m=128$} \\
& $x_{1}(t)$ & $x_{2}(t)$ & $x_{3}(t)$ & $x_{1}(t)$ & $x_{2}(t)$ & $x_{3}(t)$ \\
\hline 0.0 & 2.0000 & -1.0000 & -2.0000 & 2.0000 & -1.0000 & -2.0000 \\
0.125 & 1.7517 & -0.7517 & -1.9719 & 1.7517 & -0.7517 & -1.9719 \\
0.25 & 1.5071 & -0.5071 & -1.9431 & 1.5071 & -0.5071 & -1.9431 \\
0.375 & 1.2660 & -0.2660 & -1.9135 & 1.2660 & -0.2660 & -1.9135 \\
0.5 & 1.0287 & -0.0287 & -1.8832 & 1.0287 & -0.0287 & -1.8832 \\
0.625 & 0.7953 & 0.2047 & -1.8519 & 0.7953 & 0.2047 & -1.8519 \\
0.75 & 0.5658 & 0.4342 & -1.8196 & 0.5658 & 0.4342 & -1.8196 \\
0.875 & 0.3404 & 0.6596 & -1.7862 & 0.34040 & 0.6596 & -1.7862 \\
1.0 & 0.1193 & 0.8807 & -1.7516 & 0.1193 & 0.8807 & -1.7516 \\
\hline
\end{tabular}

\section{Conclusion}

The properties of STWS are used to solve the time-varying singular nonlinear systems. The key idea is to transform the time-varying functions into STWS. The method can be implemented using a digital computer. It occupies less memory space and consumes less computer time than the method in [9]. Illustrative examples were included to demonstrate the validity and applicability of the technique.

\section{References}

[1] K. Balachandran and K. Murugesan, Analysis of different systems via single term Walsh series method, Int. J. Comput. Math. 33 (1990), 171-180.

[2] - Analysis of nonlinear singular systems via STWS method, Int. J. Comput. Math. 36 (1990), 9-12. 


\section{STWS for singular nonlinear systems}

[3] Numerical solution of a singular nonlinear system from fluid dynamics, Int. J. Comput. Math. 38 (1991), 211-218.

[4] S. L. Campbell, Bilinear nonlinear descriptor control systems, CRSC Tech. Rep. 102386-01, Department of Mathematics, North Carolina State University, North Carolina, 1987.

[5] C. F. Chen and C.-H. Hsiao, A state-space approach to Walsh series solution of linear systems, Internat. J. Systems Sci. 6 (1975), no. 9, 833-858.

[6] - Time-domain synthesis via Walsh functions, Proc. IEE 122 (1975), 565-570.

[7] Walsh series analysis in optimal control, Internat. J. Control 21 (1975), 881-897.

[8] N. Declaris and A. Rindos, Semi state analysis of neural networks in Aplysia California, Proc. 27th MSCS, 1984, pp. 686-689.

[9] C.-H. Hsiao and W.-J. Wang, State analysis of time-varying singular nonlinear systems via Haar wavelets, Math. Comput. Simulation 51 (1999), no. 1-2, 91-100.

[10] P. Lancaster, Theory of Matrices, Academic Press, New York, 1969.

[11] F. L. Lewis, B. G. Mertzios, and W. Marszalek, Analysis of singular bilinear systems using Walsh functions, Proc. IEE-D 138 (1991), no. 2, 89-92.

[12] R. W. Newcomb, The semistate description of nonlinear time-variable circuits, IEEE Trans. Circuits and Systems 28 (1981), no. 1, 62-71.

[13] G. P. Rao, Piecewise Constant Orthogonal Functions and Their Application to Systems and Control, Lecture Notes in Control and Information Sciences, vol. 55, Springer-Verlag, Berlin, 1983.

[14] G. P. Rao, K. R. Palanisamy, and T. Srinivasan, Extension of computation beyond the limit of initial normal interval in Walsh series analysis of dynamical systems, IEEE Trans. Automat. Control 25 (1980), 317-319.

[15] P. Sannuti, Analysis and synthesis of dynamic systems via block-pulse functions, Proc. IEE 124 (1977), 569-571.

[16] N. Wiener, Cybernetics, MIT Press, Cambridge, 1965.

B. Sepehrian: Department of Applied Mathematics, Amirkabir University of Technology, Tehran 15914, Iran

E-mail address: behnamm2001ir@yahoo.com

M. Razzaghi: Department of Mathematics and Statistics, Mississippi State University, Mississippi State, MS 39762, USA; Department of Applied Mathematics, Amirkabir University of Technology, Tehran 15914, Iran

E-mail address: razzaghi@math.msstate.edu 


\section{Differential Equations \& Nonlinear Mechanics}

\section{An Open Access Journal}

\section{Editor-in-Chief}

K. Vajravelu

USA

Associate Editors

N. Bellomo

Italy

J. L. Bona

USA

J. R. Cannon

USA

S.-N. Chow

USA

B. S. Dandapat

India

E. DiBenedetto

USA

R. Finn

USA

R. L. Fosdick

USA

J. Frehse

Germany

A. Friedman

USA

R. Grimshaw

UK

J. Malek

Czech Republic

J. T. Oden

USA

R. Quintanilla

Spain

K. R. Rajagopal

USA

G. Saccomandi

Italy

Y. Shibata

Japan

Ivar Stakgold

USA

Swaroop Darbha

USA

A. Tani

Japan

S. Turek

Germany

A. Wineman

USA
Website: http://www.hindawi.com/journals/denm/

Aims and Scope

Differential equations play a central role in describing natural phenomena as well as the complex processes that arise from science and technology. Differential Equations \& Nonlinear Mechanics (DENM) will provide a forum for the modeling and analysis of nonlinear phenomena. One of the principal aims of the journal is to promote cross-fertilization between the various subdisciplines of the sciences: physics, chemistry, and biology, as well as various branches of engineering and the medical sciences.

Special efforts will be made to process the papers in a speedy and fair fashion to simultaneously ensure quality and timely publication.

DENM will publish original research papers that are devoted to modeling, analysis, and computational techniques. In addition to original full-length papers, DENM will also publish authoritative and informative review articles devoted to various aspects of ordinary and partial differential equations and their applications to sciences, engineering, and medicine.

\section{Open Access Support}

The Open Access movement is a relatively recent development in academic publishing. It proposes a new business model for academic publishing that enables immediate, worldwide, barrier-free, open access to the full text of research articles for the best interests of the scientific community. All interested readers can read, download, and/or print any Open Access articles without requiring a subscription to the journal in which these articles are published.

In this Open Access model, the publication cost should be covered by the author's institution or research funds. These Open Access charges replace subscription charges and allow the publishers to give the published material away for free to all interested online visitors.

\section{Instructions for Authors}

Original articles are invited and should be submitted through the DENM manuscript tracking system at http://www.mstracking.com/ denm/. Only pdf files are accepted. If, for some reason, submission through the manuscript tracking system is not possible, you can contact denm.support@hindawi.com.

Hindawi Publishing Corporation

410 Park Avenue, 15th Floor, \#287 pmb, New York, NY 10022, USA
HINDAWI 


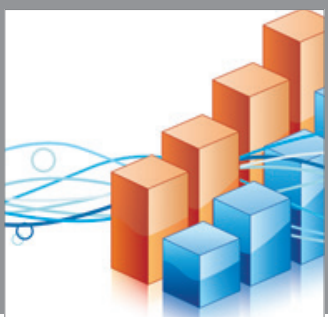

Advances in

Operations Research

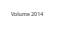

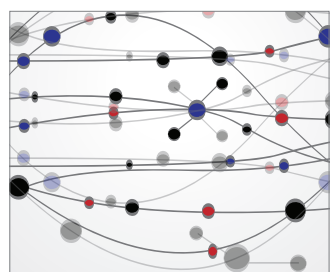

\section{The Scientific} World Journal
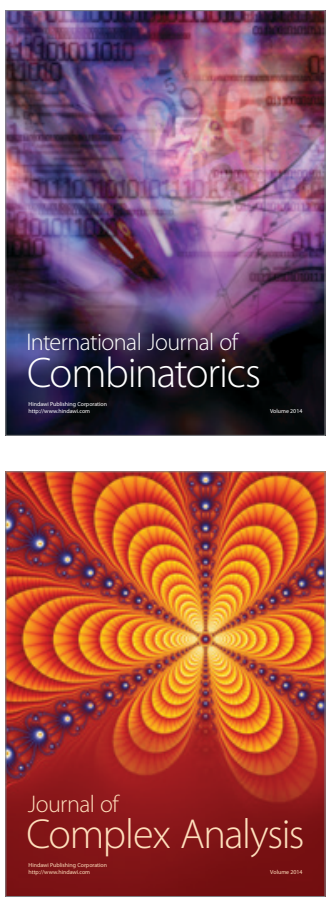

International Journal of

Mathematics and

Mathematical

Sciences
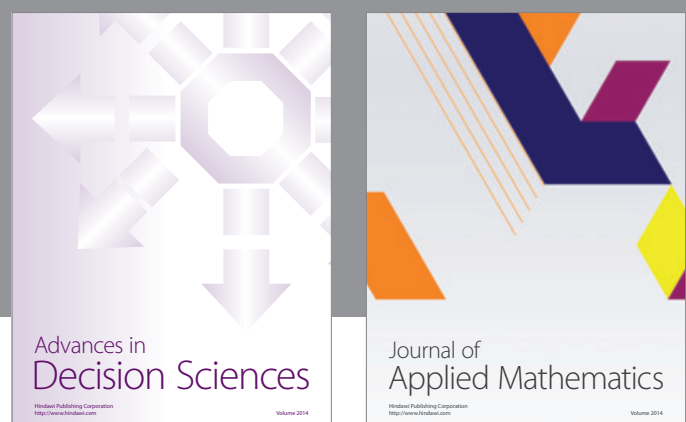

Journal of

Applied Mathematics
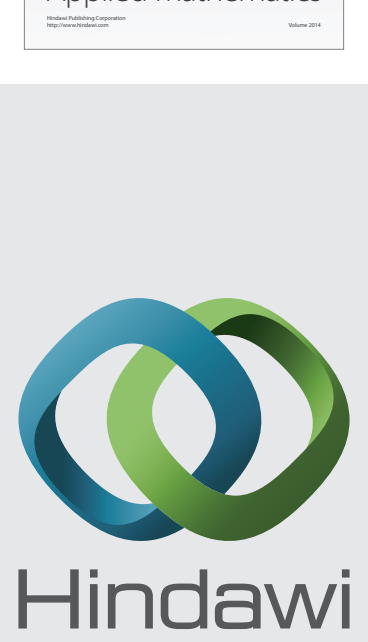

Submit your manuscripts at http://www.hindawi.com
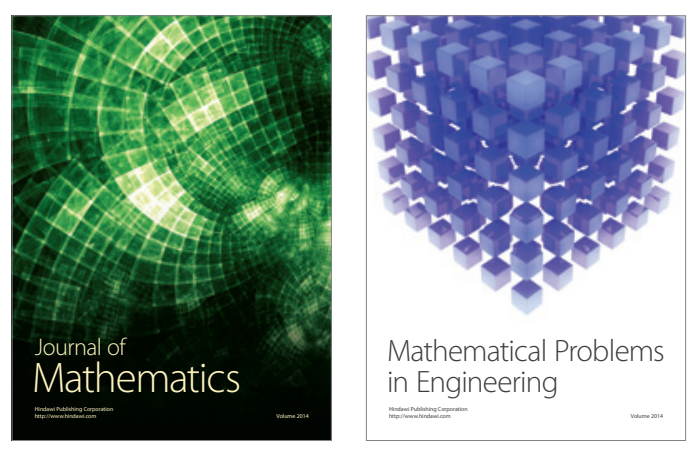

Mathematical Problems in Engineering
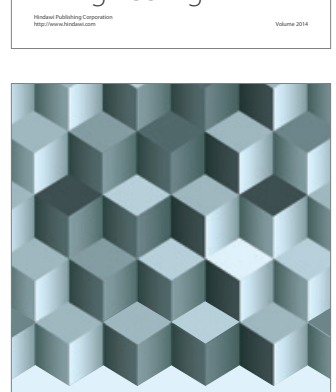

Journal of

Function Spaces
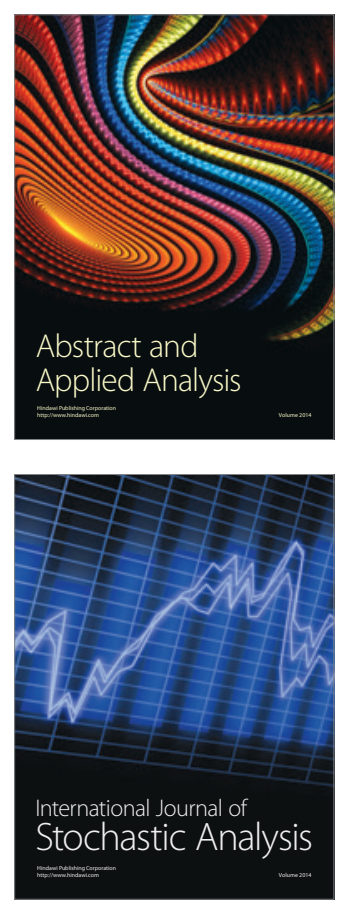

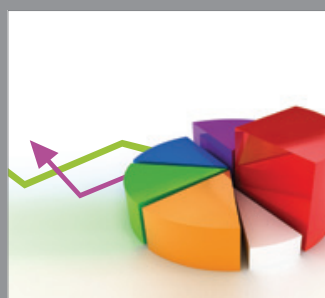

ournal of

Probability and Statistics

Promensencen
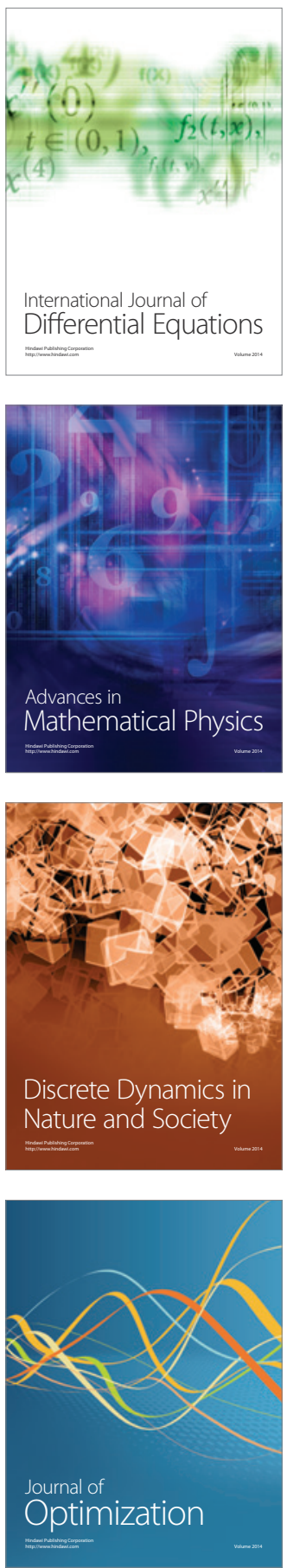